\title{
A low-cost programmable pulse generator for physiology and behavior
}

\section{Joshua I. Sanders * and Adam Kepecs}

Neuroscience, Cold Spring Harbor Laboratory, Kepecs Lab, Cold Spring Harbor, NY, USA

\section{Edited by:}

Laura Ballerini, University of Trieste,

Italy

Reviewed by:

Domenico Caputo, La Sapienza

University, Italy

Hun-Kuk Park, Kyung Hee

University, South Korea

*Correspondence:

Joshua I. Sanders, Neuroscience,

Cold Spring Harbor Laboratory,

Kepecs Lab, Cold Spring Harbor, 1

Bungtown Road, NY 11724, USA

e-mail:sanders@

cshl.edu

\begin{abstract}
Precisely timed experimental manipulations of the brain and its sensory environment are often employed to reveal principles of brain function. While complex and reliable pulse trains for temporal stimulus control can be generated with commercial instruments, contemporary options remain expensive and proprietary. We have developed Pulse $\mathrm{Pal}$, an open source device that allows users to create and trigger software-defined trains of voltage pulses with high temporal precision. Here we describe Pulse Pal's circuitry and firmware, and characterize its precision and reliability. In addition, we supply online documentation with instructions for assembling, testing and installing Pulse Pal. While the device can be operated as a stand-alone instrument, we also provide application programming interfaces in several programming languages. As an inexpensive, flexible and open solution for temporal control, we anticipate that Pulse Pal will be used to address a wide range of instrumentation timing challenges in neuroscience research.
\end{abstract}

Keywords: Pulse Pal, stimulator, open source, optogenetics, maple, arduino, pulse generator, timing

\section{INTRODUCTION}

Patterned voltage pulse trains are commonly used in neuroscience research to precisely control stimulus isolators (Flaherty and Graybiel, 1994; Bisley et al., 2001; Cohen and Newsome, 2004; Histed et al., 2009), light sources for optogenetic manipulations (Boyden et al., 2005; Cardin et al., 2009), sensory stimuli (Soto-Faraco et al., 2002), and to synchronize events between instruments (Nikolic et al., 2009). Pulse trains can also be also triggered by a particular experimental contingency, providing closed-loop feedback at low latency (Girardeau et al., 2009; Venkatraman et al., 2009; Berényi et al., 2012; Newman et al., 2013). Laboratory instruments specialized for these purposes are commercially available, for example Master 8 (AMPI), PSG-2 (ISSI), Pulsemaster A300 (WPI), BPG1 (Bak Electronics), StimPulse PGM (FHC Inc.) and Multistim 3800 (A-M Systems). Commercial solutions have been widely adopted, however their cost is a constraint in research and educational settings with limited funding. As proprietary instruments, researchers are also unable to add hardware or software features that would suit their unique needs: for instance, to implement a custom set of triggering rules in firmware, or to develop an interface to the device in a desired programming language. This flexibility can be especially beneficial for experimental design in systems neuroscience, where integration of custom instrumentation is frequently employed to measure and control behavior (Brunton et al., 2013), acquire neural data (Yamamoto and Wilson, 2008; Karlsson and Frank, 2009) and to stimulate the brain both electrically (O'Doherty et al., 2009) and optically (O'Connor et al., 2013).
To address these needs, we developed Pulse Pal (Figure 1), an open source pulse train generator costing $\sim \$ 210$ (US) in easily obtained parts, with essential functionality comparable to commercial stimulators.

\section{SYSTEM DESIGN HARDWARE}

Pulse Pal was designed to be assembled at a laboratory soldering bench in approximately $1 \mathrm{~h}$ with minimal tools: a soldering iron, solder, a miniature Phillips head screw driver and a 4-40 tap. We provide instructions for ordering the necessary parts, assembling the device and programming firmware on the Pulse Pal wiki ${ }^{1}$. Hardware design files, drivers, firmware, and software interfaces to the device in MATLAB, Python and C++ are provided in a public repository. ${ }^{2}$ The assembled device and example pulse trains demonstrating key features are shown in Figure 1.

Pulse Pal's essential triggering and stimulation circuit for a single trigger and analog output channel are shown in Figure 2. Pulse Pal passes incoming trigger logic signals through an optocoupler IC to protect microcontroller input pins and reduce potential for ground loops. Trigger signals are then read by Pulse Pal's ARM Cortex M3 microcontroller (STM32F103RBT6, ST microsystems) provided as part of the open source Maple microcontroller platform (LeafLabs). The microcontroller generates analog waveforms by controlling an external 4-channel digital to analog converter (DAC) IC (MAX500ACPE+, Maxim

\footnotetext{
${ }^{1}$ https://sites.google.com/site/pulsepalwiki/home

${ }^{2}$ https://github.com/PulsePal/PulsePal
} 

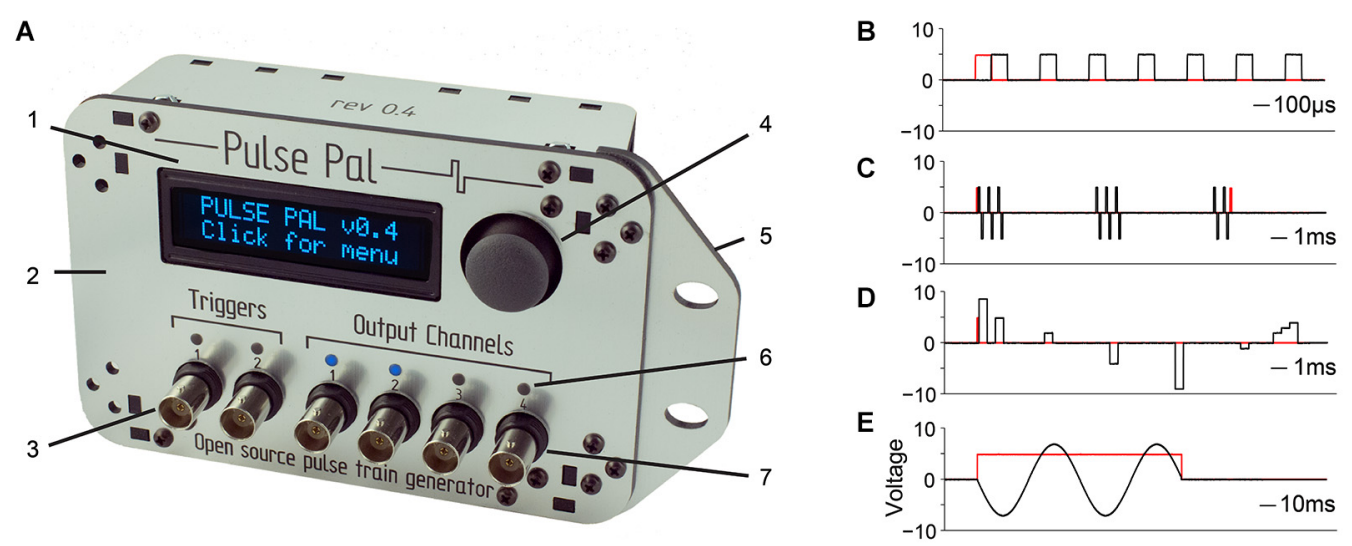

FIGURE 1 | Pulse Pal is a programmable pulse train generator. (A) Pulse Pal front view, illustrating front panel features. 1: High contrast oLED screen permits programming with thumb joystick for stand-alone use. 2: Custom laser-cut acrylic enclosure. 3: Two optically isolated digital trigger channels. 4: Thumb joystick. 5: Rack-mount wing. 6: Channel activity indicators illuminate when channel voltage is not the set resting voltage (i.e., during a pulse). 7: Each of four analog output channels can be programmed with independent pulse trains and linked to either trigger channel. (B-E) Example pulse trains in black, acquired with an oscilloscope (see methods). Trigger voltage traces are shown in red. (B) A Pulse Pal output channel configured to deliver a train of $5 \mathrm{~V}, 100 \mu \mathrm{s}$ square pulses with $200 \mu \mathrm{s}$ intervals. (C) A train of biphasic +/-5 V $100 \mu \mathrm{s}$ pulses, gated programmatically to produce pulse bursts. Trigger channel mode set to "toggle" aborts the ongoing pulse train in mid-burst when a second pulse arrives. (D) A train of $500 \mu \mathrm{s}$ pulses with custom onset times and voltages. Pulses with consecutive onset times merge to form more complex waveforms (right). (E) A train of consecutive $100 \mu \mathrm{s}$ pulses, whose voltages and onset times were configured to generate one period of a sine waveform. The output channel uses "loop mode", to repeat the sine waveform until a parametrically specified pulse train end. The trigger channel mode was set to "pulse gated" mode, to abort the pulse train when its voltage returned low.
Integrated Products), configured with bipolar output circuitry as specified in figure 9 of the MAX500 datasheet. This output circuitry consists of an Op Amp (TL084ACN, Texas Instruments) and two $10 \mathrm{k}$ resistors $(\mathrm{R} 3, \mathrm{R} 4)$ that divide the $\mathrm{DAC}$ reference voltage, collectively providing output voltages in the range of -10 to $+10 \mathrm{~V}$ from each (otherwise unipolar) DAC output channel. A capacitor (C1) was added across each amplifier to suppress voltage transient overshoot. Voltage instructions are sent to the DAC over an $18 \mathrm{MHz}$ hardware serial bus. For bipolar operation in the range of -10 to $+10 \mathrm{~V}$, the DAC requires power supplied at $+/-12$ VDC. This supply is derived from Maple's USB power supply with an integrated DC voltage converter (CC3-512DF$\mathrm{E}$, TDK Lambda). To set the range of the DAC to $+/-10 \mathrm{~V}$, a separate $10 \mathrm{~V}$ reference voltage is provided to the DAC from the $+12 \mathrm{~V}$ supply, using a linear voltage regulator (L78S10CV, ST Microsystems).

Further circuitry (not shown in Figure 2) was added to equip Pulse Pal for stand-alone operation. An oLED character display (NHD-0216KZW-AB5, Newhaven Display) and a two-axis pushbutton thumb joystick (802-30110A, P3 America) are used as an interface to program each channel's parameters and test-trigger the device from a menu tree implemented in firmware. To retain parameters across power cycles, we added an external EEPROM IC (on a separate $9 \mathrm{MHz}$ serial bus to accommodate the chip's lower clock speed constraint; 25LC640A-I/P, Microchip Technology). An LED above each channel was added to indicate when the channel's voltage is set to a value different from its programmed resting voltage (i.e., the channel is delivering a pulse). The complete schematic and circuit board layout are provided in the
Pulse Pal repository, as files for Eagle printed circuit board (PCB) software (CadSoft) and as GERBER files for PCB manufacture.

\section{SOFTWARE}

The ARM processor that subserves Pulse Pal was programmed with custom firmware, written in the LeafLabs derivative of the Arduino language - a $\mathrm{C}++$ based programming language for AVR and ARM microcontrollers. Pulse Pal's firmware was programmed to execute its main loop every $50 \mu \mathrm{s}$ when delivering pulse trains. Loop execution is triggered by a hardware timer, provided as an internal feature of the microcontroller. On each loop cycle, the microcontroller updates the DAC, reads triggerchannel logic and any single-byte USB serial instructions, computes the logic of the current cycle's voltage transitions, and adjusts indicator LED status. USB serial instructions employ a system of single byte op-codes, enabling a software client to program and trigger Pulse Pal, abort ongoing stimulation, set fixed voltages for output channels, or set logic values of Maple's I/O lines for debugging. To distinguish it from subsequent updates, the firmware version used to acquire performance data for the present publication is provided in a dedicated folder in the code repository.

Pulse Pal is programmed either using its thumb joystick interface or via USB by setting channel parameters (indicated below by their cross-platform syntax in italic, and illustrated in Figure 3 for output channels). A single parameter for each trigger channel, TriggerMode, controls how it interprets incoming logic. Three trigger modes are provided: "normal", "toggle" and "pulse gated". In normal mode, an incoming logic pulse triggers 


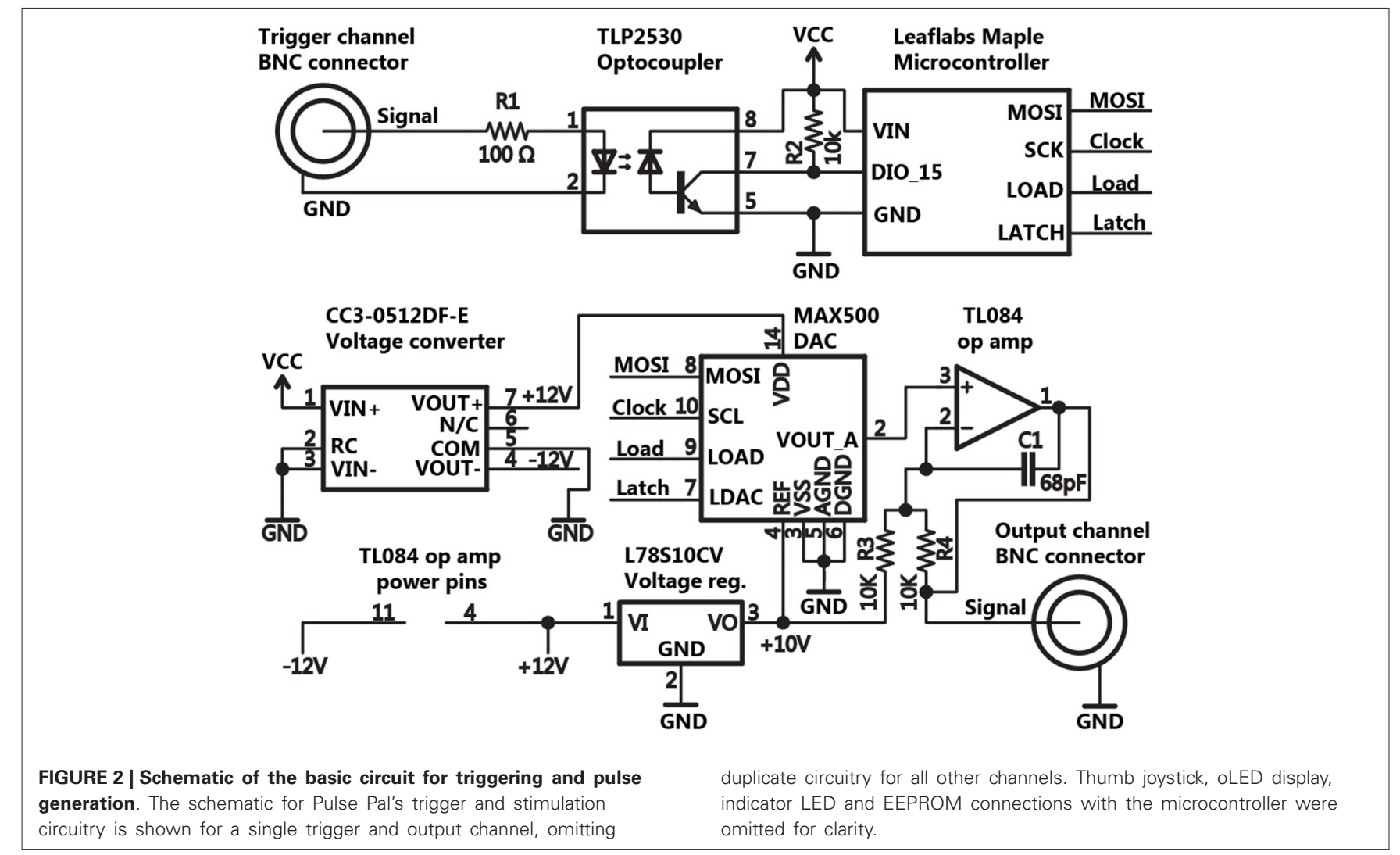

all linked output channels, but subsequent triggers are ignored during playback. In toggle mode, subsequent triggers terminate ongoing pulse trains on linked output channels. In pulse gated mode, pulse trains are triggered by a low to high logic transition on the trigger channel, and terminated by the subsequent high to low transition if it occurs during playback.

The stimulus parameters of each output channel can be independently programmed. Output channels can deliver either parametric pulse trains or custom pulse trains, for which each pulse has a specified onset time and voltage. Pulse shape and frequency are defined by 7 parameters: IsBiphasic ( 0 if pulses are monophasic, 1 if biphasic), PhaselVoltage (voltage of the first phase, $-10 \mathrm{~V}$ to $+10 \mathrm{~V}$ ), Phase2Voltage (same range), Phase1Duration (duration of the first phase, $0.1 \mathrm{~s}$ to $3600 \mathrm{~s}$ ), InterPhaseInterval (the time between phases of a biphasic pulse), Phase2Duration, and InterPulseInterval (time between pulses). Pulse trains are defined by an additional 4 parameters: BurstDuration (time during which the underlying pulse train is gated "on") BurstInterval (a period alternating with BurstDuration during which the pulse train is gated "off"), PulseTrainDelay (time between trigger and pulse train onset), and PulseTrainDuration (duration of the pulse train). Two custom pulse trains of up to 1,000 pulses each can be defined, where the user specifies the onset time and voltage of each pulse. Use of a custom train on an output channel is selected by setting the channel's CustomTrainID parameter to a non-zero value (1 or 2, specifying which train). For custom trains, two additional parameters are configurable: CustomTrainTarget (for onset times and voltages; 0 if these refer to pulses, 1 if these refer to bursts of pulses), and CustomTrainLoop (0 if pulse train ends after final pulse defined, 1 if pulse train loops from trigger until the value of PulseTrainDuration). Each output channel has three additional settings: LinkTriggerChannel1, LinkTriggerChannel2 and RestingVoltage. The first two of these parameters specify which trigger channels control the output channel. The third specifies the output channel's resting voltage between pulse phases, pulses and pulse trains $(0 \mathrm{~V}$ by default).

Design files for a device enclosure that can be laser cut from a single sheet of $30.48 \times 30.48 \mathrm{~cm}\left(12^{\prime \prime} \times 12^{\prime \prime}\right)$ acrylic are provided in the repository. Raster-engraved text in the design indicates channel and USB port identities. Light pipes (PLP2, Bivar) press into holes above each channel, routing light from indicator LEDs on the circuit board to the enclosure surface. The enclosure attaches to the circuit board with screws fastened to threaded circuit board stand-offs, and contains a removable wing for attaching the device to a server rack (shown in Figure 1A).

\section{MEASURES OF RELIABILITY AND PRECISION}

To validate Pulse Pal as a practical solution for stimulus control, we tested the precision and reliability of the shortest pulses the device can process on both trigger and output channels, and several other properties relevant for neurophysiology research. All tests were performed on a single Pulse Pal device, connected to a controlling computer (Macbook Pro, Apple). 


\section{Output channel parameters}

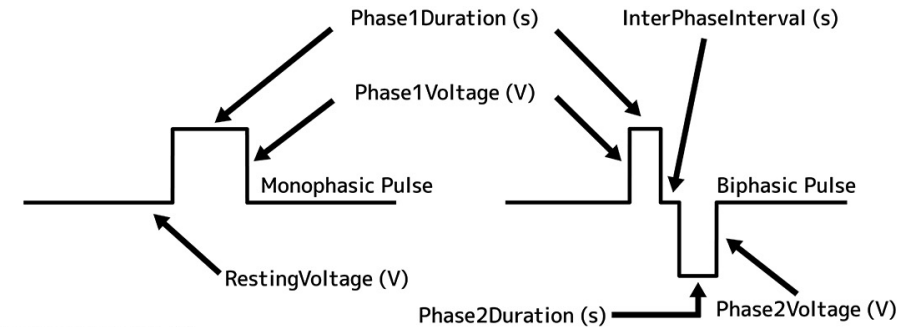

InterPulselnterval (s)

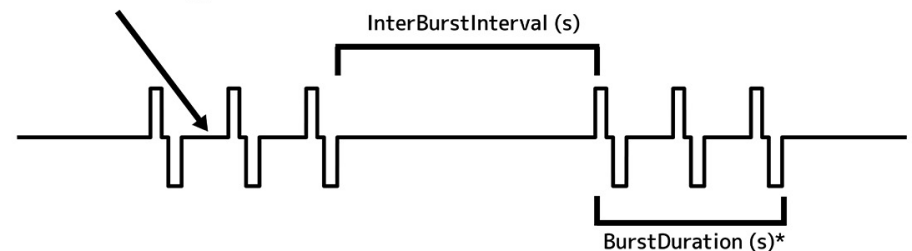

PulseTrainDuration (s)

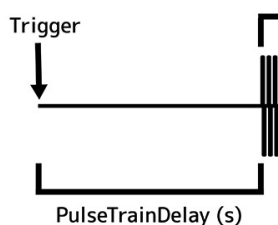

\section{Pulse type, linking and custom trains}

IsBiphasic

0 : channel uses monophasic pulses

1: channel uses biphasic pulses

\section{LinkTriggerChannel1}

0 : channel ignores trigger channel 1

1: channel triggered by trigger ch1

LinkTriggerChannel2

0 : channel ignores trigger channel 2

1: channel triggered by trigger ch2

CustomTrainID

0 : channel uses parametric pulse train

1: channel uses custom pulse train 1

2: channel uses custom pulse train 2

CustomTrainTarget

0 : custom times define pulse onsets

1: custom times define burst onsets

CustomTrainLoop

$0:$ custom train ends after last pulse

1: train loops until PulseTrainDuration

* Set to 0 for continuous pulse train (no bursts)

FIGURE 3 | Illustration of output channel parameters

While output channels are updated once per execution of the microcontroller's $50 \mu$ s main loop, the shortest configurable pulse is restricted to $100 \mu \mathrm{s}$ (to ensure that the smallest output channel pulse can also reliably trigger the device). To measure the precision of a $100 \mu$ s pulse, we programmed Pulse Pal to deliver a train of three $100 \mu \mathrm{s}$ pulses, separated by $100 \mu \mathrm{s}$ intervals on all 4 output channels each time a software trigger was detected. Pulse Pal's first output channel was connected to a digital oscilloscope (DS1102D, Rigol). Pulse Pal was then software-triggered 100,000 times over $24 \mathrm{~h}$ by a custom test script written in MATLAB r2013a (Mathworks) on the controlling computer. After each trigger event, the resulting waveform was returned from the oscilloscope to the computer. Pulse Pal generated a unique waveform of three pulses after each trigger, demonstrating high software trigger reliability. The first 100 pulse trains are shown superimposed in Figure $4 \mathrm{~A}$, aligned to first pulse onset to demonstrate pulse jitter. The pulse widths of all 300,000 pulses are shown in Figure 4B. Cycle widths ranged from $96.9 \mu \mathrm{s}$ to $102.9 \mu \mathrm{s}$, and $99.97 \%$ of pulses were within $3 \mu \mathrm{s}$ of $100 \mu \mathrm{s}$.

Next, we measured clock drift, to ensure that the timing variability we observed in $100 \mu$ s-long pulses did not propagate. We programmed Pulse Pal to deliver a single $10 \mathrm{~s}$ pulse (spanning 200,000 microcontroller loop cycles) when triggered. We captured the resulting waveform on 10,000 trials with an analog capture device (NI USB-6210, National Instruments), sampled at $100 \mathrm{kHz} .100$ example pulse waveforms are shown in Figure 4C, and all 10,000 pulse widths are shown in Figure 4D. All pulses measured either $9.99998 \mathrm{~s}$ or $9.99997 \mathrm{~s}$, corresponding to a consistent clock drift of $3 \mu \mathrm{s} / \mathrm{s}$ with respect to the NI USB-6210 clock.

To measure pulse train latency and trigger channel reliability, we connected two Pulse Pals in series. The first was softtriggered by the computer on each of 100,000 trials. It generated a single $5 \mathrm{~V}, 100 \mu \mathrm{s}$ square pulse simultaneously on two output channels-one delivered to the first trigger channel of the second Pulse Pal, and the other to an oscilloscope (see Figure $4 \mathbf{H}$ for a separate experiment demonstrating the simultaneity of these pulses). The second Pulse Pal generated a single $100 \mu$ s pulse on each output channel when its first trigger channel was triggered, which was captured from one output channel by a separate oscilloscope channel. In Figure 4E, 100 trials are shown. Trigger pulses from the first (triggering) Pulse Pal are shown in red, and pulses from the second Pulse Pal in black. All 100,000 pulses captured were unique waveforms, indicating high hardware trigger reliability. Output channel latency for all 100,000 trials ranged relatively uniformly between 91.0 and $146.7 \mu \mathrm{s}$ (Figure 4F).

Next we sought to determine whether channel noise was low enough that a single bit DAC increment produced a non-overlapping change in voltage. Since the MAX500ACPE+ DAC that drives Pulse Pal's output channels has 8-bits of precision mapped across a $20 \mathrm{~V}$ range $(-10 \mathrm{~V}$ to $+10 \mathrm{~V})$, its least significant bit (LSB) increments the channel voltage by $78.1 \mathrm{mV}$. Therefore we set up PulsePal to trigger two $78.1 \mathrm{mV}$ pulses 100 times (shown in Figure 4G). Fluctuations in voltage on individual trials ranged as much as 
A

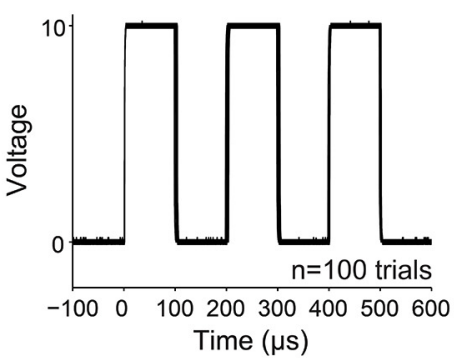

C

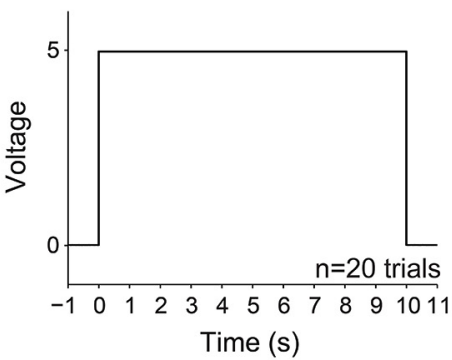

E

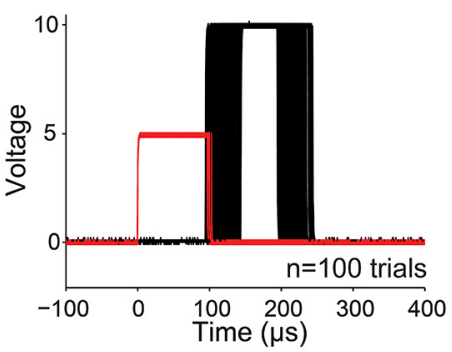

B

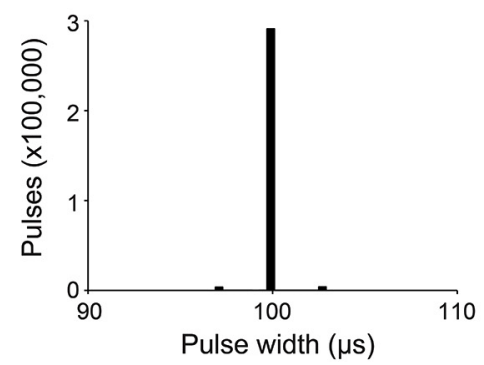

D

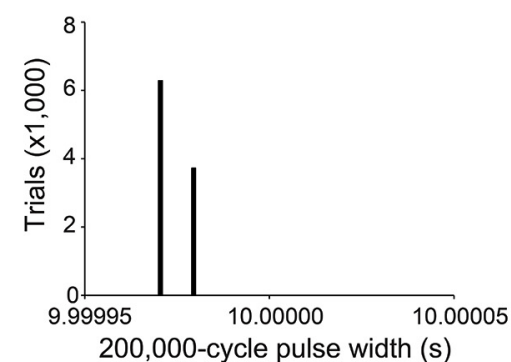

$\mathbf{F}$

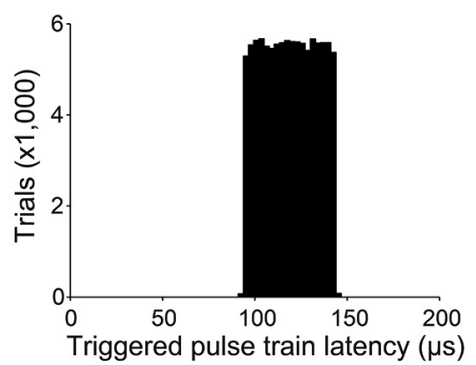

G

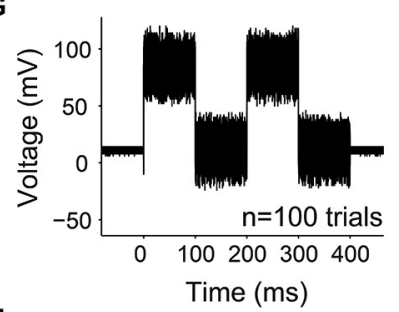

H
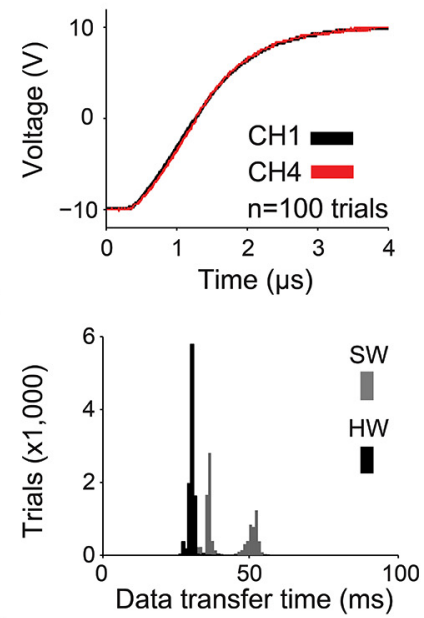

$\mathrm{J}$

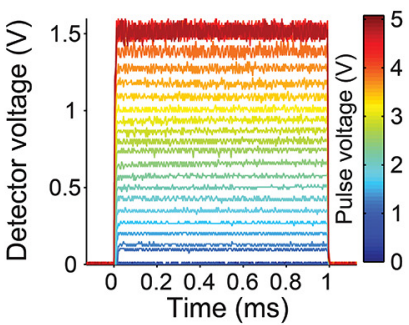

FIGURE 4 | Measurements of precision and reliability. (A-B) For a train of three $100 \mu \mathrm{s}$ pulses with $100 \mu \mathrm{s}$ pulse intervals: (A) the first 100 waveforms captured with the oscilloscope are shown superimposed, and (B) distribution of pulse widths measured from 100,000 3-pulse trains, captured as in (A). (C-D) For a train of a single 10 s pulse: (C) waveforms from the first 20 trials and (D) 10,000 pulse widths. (E) The latency of a pulse train of one $10 \mathrm{~V}, 100 \mu \mathrm{s}$ pulse captured from an output channel (shown in black for 100 trials) was measured with respect to a $5 \mathrm{~V}, 100 \mu \mathrm{s}$ pulse delivered to a linked trigger channel (shown in red). (F) Distribution of pulse train latencies for 100,000 trials. (G) 100 superimposed $78.1 \mathrm{mV}$ pulses, showing the smallest possible increment of the digital to analog converter and channel noise caused by digital feed-through from the SPI bus. (H) Simultaneous and rapid settling of the voltage on channels 1 and 4 when delivering $a+10 \mathrm{~V}$ pulse from a resting voltage of $-10 \mathrm{~V}$. (I) USB transfer time is shown for a 5,006 byte message containing pulse times and voltages for a 1,000-pulse custom train. Transfer time was measured with hardware (HW, black; using firmware modified to indicate transmission start and end with a voltage pulse) and software (SW, gray; using the controlling computer's clock). (J) $1 \mathrm{~ms}$ pulses of light, produced by controlling a blue diode laser with Pulse Pal, converted to voltage with an Si transimpedence amplified photodetector (PDA10A, ThorLabs), and captured with an oscilloscope. Single traces are shown for voltage pulses ranging from $78 \mathrm{mV}$ to $5 \mathrm{~V}$ in amplitude.
$8 \mathrm{mV}$ about the mean in the $100 \mathrm{~ms}$ interval prior to pulse train onset, and as much as $65 \mathrm{mV}$ about the mean during the first $100 \mathrm{~ms}$ pulse. The increased noise during playback was mostly attributable to digital feed-through from the SPI channels controlling the DAC (data not shown), but remained significantly less than the DAC's minimum voltage increment. While several board layout and circuit modifications could be implemented by researchers wishing to further reduce digital feed-through (for instance, by optical isolation of the SPI bus), Pulse Pal can exploit the full bit- width of its DAC in its present form, rendering it useful for many control applications in neuroscience instrumentation. The resting voltage of the output channel was programmatically set to $0 \mathrm{~V}$, but was measured in this experiment to be $10.55 \mathrm{mV}$ (within the $15 \mathrm{mV}$ "zero code error" specified for the MAX500ACPE+ DAC in its datasheet), indicating that an offset from the $0 \mathrm{~V}$ set-point was present but slight.

In experiments with precisely timed events, it is useful to produce signals that occur simultaneously. Therefore we measured 
the simultaneity of output channel updates by comparing pulses triggered on the first and last output channels. We set the first and fourth output channels to a resting voltage of $-10 \mathrm{~V}$, delivered $100+10 \mathrm{~V}$ pulses, and captured the rising waveform of each pulse with the oscilloscope (Figure $4 \mathbf{H}$ ). On all trials, the output voltage on both channels settled within $100 \mathrm{mV}$ of $+10 \mathrm{~V}$ after $3.5 \mu \mathrm{s}$. This measurement also confirmed that DAC and output amplifier slew rates were fast enough to produce 100 us pulses useful for most applications in neuroscience research.

In many experiments, pulse train parameters and timing data must be updated rapidly in response to recently acquired information. Since the same microcontroller controls pulse timing and USB communication, Pulse Pal cannot be updated while a pulse train is being delivered. Therefore we sought to ensure that updates could be achieved rapidly between experimental trials. We measured the USB data transfer speed by sending a 1000-pulse train (5006 bytes) 100 times, from the Pulse Pal MATLAB client to Pulse Pal. For a measure of the hardware transfer speed without client-side software overhead, Pulse Pal's firmware was modified to indicate the start of data transfer by setting output channel $1-5 \mathrm{~V}$, and the end of data transfer by returning channel $1-0 \mathrm{~V}$. The resulting pulse was captured by the oscilloscope on each trial. The client-side transfer time was separately measured for the blocking MATLAB serial fwrite command, by flanking it with tic and toc commands. Transfers completed in 26-35 ms (averaging $171 \mathrm{~KB} / \mathrm{s}$ ), while client-side overhead cost an additional $12 \mathrm{~ms}$ on average (Figure 4I). Consistent with this transfer speed measurement, a separate transfer updating all of Pulse Pal's channel parameters for all channels (163 bytes) completed on the hardware side in less than $1 \mathrm{~ms}$ (data not shown).

Finally, we sought to verify Pulse Pal's suitability for precise optical control in optogenetic experiments, by using it to control a $447 \mathrm{~nm}$ diode laser's timing and intensity. We connected the laser (BML447-50FLD, Lasermate Group) through an optical fiber (M31L02, ThorLabs) to a silicon transimpedence amplified photodetector (PDA10A, ThorLabs), delivered 1 ms pulses from a Pulse Pal output channel to the laser power supply's analog input, and captured the resulting waveforms with an oscilloscope (DS1102D, Rigol). Pulses ranged in amplitude from $78 \mathrm{mV}$ to $5.0 \mathrm{~V}$ in $78 \mathrm{mV}$ increments. In Figure 4J, the single traces captured for each voltage are shown superimposed, skipping every second voltage for clarity. Pulse Pal elicited precisely gated pulses of light from the laser, with programmatic (albeit slightly nonlinear) control of light intensity.

\section{APPLICATIONS LIGHT TIMING AND INTENSITY CONTROL FOR OPTOGENETICS}

Pulse Pal was originally developed in a laboratory setting, to provide an intuitive and affordable way to achieve fine temporal control in optogenetics experiments (Pi et al., 2013). In these studies, Pulse Pal was used to control a laser coupled to an optical fiber as in Figure 4J, providing precisely timed pulse trains to photostimulate specific classes of interneurons. In this role, Pulse Pal provides a simple and open alternative to commercial pulse train generators (e.g., Master 8 (AMPI), PSG-2 (ISSI), Pulsemaster A300 (WPI), BPG-1 (Bak Electronics), StimPulse PGM (FHC Inc.) and Multistim 3800 (A-M Systems).

\section{SENSORY PATTERN GENERATION WITH LOW LATENCY GATING}

In the same research study, Pulse Pal doubled as a programmable waveform generator, providing simple, low-latency acoustic stimuli for a $\mathrm{Go} / \mathrm{No}-\mathrm{Go}$ perceptual decision task. In this application, each output channel directly drove a separate amplified speaker. Beyond the simple cues used in these experiments, temporally patterned auditory and visual pulse stimuli are often used to study the algorithmic basis for human and animal decision making. A binaural Poisson click stream (Sanders and Kepecs, 2012; Brunton et al., 2013) can be generated using Pulse Pal's custom pulse trains, where a 100 us, $1 \mathrm{~V}$ pulse delivered to an amplified headphone speaker generates a precisely timed audible click. For visual stimuli, each channel can be configured to produce precisely timed visual flashes (Zylberberg et al., 2012), by gating a commercial LED driver (e.g., BuckPuck, LED Dynamics). Thus, the stimuli can be triggered and stopped with much lower latency and higher temporal precision than a commercial sound card or computer video display (Kleiner et al., 2007). For sensory decision making experiments that require fine temporal control, Pulse Pal provides a simple and open alternative to custom instrumentation.

\section{GENERAL ANALOG CONTROL OF LABORATORY INSTRUMENTS}

Several neuroscience instruments use analog signals as an interface to control device parameters. Some examples are galvanometer mirrors for laser scanning stimulation (e.g., GVSM002, Thor Labs), and monochromators to measure spectral tuning in optogenetics (e.g., Polychrome V, Till Photonics). Programmatic control of unipolar voltage can be accomplished inexpensively in some cases with microcontroller platforms (e.g., Arduino) or low-cost automation devices (e.g., U3, LabJack). However, many devices (including the two listed above) require control voltages in the industry standard range of $-10 \mathrm{~V}$ to $+10 \mathrm{~V}$, necessitating expensive, proprietary computer hardware (e.g., NI PCIe-6323, National Instruments). For these applications, Pulse Pal provides an inexpensive way to achieve analog control.

\section{CLOSED-LOOP FEEDBACK IN ELECTROPHYSIOLOGY}

As a temporal control tool, Pulse Pal complements a growing array of open source acquisition tools for neuroscience research, which have become available in recent years. These range from electrophysiology acquisition systems (Rolston et al., 2009; Voigts et al., 2013a) to electrode interface devices (Voigts et al., 2013b) and data acquisition software tools (Brainard, 1997; Pologruto et al., 2003; Englitz et al., 2013; Campagnola et al., 2014). Pulse Pal has been formally integrated into the software for one of these tools, the Open Ephys electrophysiology acquisition system, ${ }^{3}$ where it is provided as one method for low latency closed loop feedback.

\footnotetext{
${ }^{3}$ www.open-ephys.org
} 


\section{GENERAL DISCUSSION}

For our research, we required a pulse generator with high precision at timescales relevant for alignment of stimulation events to action potentials (pulse time jitter at least a factor of 10 less than an action potential width; Figures 4A,B), low clock drift (Figures 4C,D), first pulse latency (Figures 4E,F) comparable to short mammalian action potentials (Kandel et al., 2000) and high reliability (100\% of 300,000 soft triggers, $100 \%$ of 100,000 $100 \mu$ s trigger pulses). In developing Pulse Pal, we recognized that simplified control of voltage pulse timing is a general need, and expanded the project's scope to meet five additional design objectives: low material cost (\$210 USD), stand-alone functionality (Figure 1), bench-side assembly with common tools (see illustrated guide on wiki), support for common computing platforms and programming languages (WinXP, Win7, OSX, Ubuntu 14.04; MATLAB, C++, Python) and comprehensive online documentation.

In fulfilling these objectives, Pulse Pal provides a general resource for precise temporal control of stimulation and environmental cues in the laboratory. It encapsulates the problem of temporal pattern generation for many applications in physiology and psychophysics, where in lieu of commercial instrumentation, this control problem had often been addressed ad-hoc by writing custom software for microcontrollers (da Silva Pinto et al., 2011; Weick et al., 2011; Bugaj et al., 2013; Haikala et al., 2013; Ohayon et al., 2013; Smear et al., 2013; Inagaki et al., 2014; Klapoetke et al., 2014).

Pulse Pal's parametric approach to stimulation features trigger logic rules and stimulus pattern motifs commonly implemented in neuroscience research, however custom applications may require the device to perform less common functions. Unlike its commercial counterparts, Pulse Pal's firmware is provided in the public domain with an open source license. To facilitate access, the firmware was written in the Arduino language ${ }^{4}$ - a reduced set of $\mathrm{C}++$ syntax with extensive online documentation for developers who lack a programming background. The adaptation of Arduino for Pulse Pal's microcontroller platform ${ }^{5}$ exposes further functionality specific to the ARM Cortex M3 microcontroller. We anticipate that this lower barrier to entry will be exploited by researchers using Pulse Pal's hardware, firmware and software as a starting point for tailored applications beyond its present niche.

\section{ACKNOWLEDGMENTS}

The following people are gratefully acknowledged for their contributions to Pulse Pal: Josh Siegle for contributing initial work on the $\mathrm{C}++$ client and providing integration with the Open Ephys GUI, and Duda Kvitsiani, Ebru Demir, Hyun-Jae Pi, Balázs Hangya, Junya Hirokawa, Alex Vaughan, Uri Livneh, Onyekachi Odemene, Matthew Kaufman, Brittany Cazakoff, Steven Shea, Rob Campbell, Ankit Sethi, Ofer Yizhar, Jeffrey Erlich and Gabriella Nyitrai for feedback during beta testing. Also, we are thankful to the LeafLabs team and members of the LeafLabs forum, especially users gbulmer, mbolivar, siy and ala32 for

${ }^{4}$ www.arduino.cc

${ }^{5}$ www.leaflabs.com frequent assistance with the Maple platform. This research was supported by grants from the US National Institutes of Health (R01NS07553, R01MH097061) and the McKnight Foundation.

\section{REFERENCES}

Berényi, A., Belluscio, M., Mao, D., and Buzsáki, G. (2012). Closed-loop control of epilepsy by transcranial electrical stimulation. Science 337, 735-737. doi: 10. $1126 /$ science. 1223154

Bisley, J. W., Zaksas, D., and Pasternak, T. (2001). Microstimulation of cortical area MT affects performance on a visual working memory task. J. Neurophysiol. 85, 187-196.

Boyden, E. S., Zhang, F., Bamberg, E., Nagel, G., and Deisseroth, K. (2005). Millisecond-timescale, genetically targeted optical control of neural activity. Nat. Neurosci. 8, 1263-1268. doi: 10.1038/nn1525

Brainard, D. H. (1997). The psychophysics toolbox. Spat. Vis. 10, 433-436. doi: 10. $1163 / 156856897 x 00357$

Brunton, B. W., Botvinick, M. M., and Brody, C. D. (2013). Rats and humans can optimally accumulate evidence for decision-making. Science 340, 95-98. doi: 10. 1126/science. 1233912

Bugaj, L. J., Choksi, A. T., Mesuda, C. K., Kane, R. S., and Schaffer, D. V. (2013). Optogenetic protein clustering and signaling activation in mammalian cells. Nat. Methods 10, 249-252. doi: 10.1038/nmeth.2360

Campagnola, L., Kratz, M. B., and Manis, P. B. (2014). ACQ5: an open-source software platform for data acquisition and analysis in neurophysiology research. Front. Neuroinform. 8:3. doi: 10.3389/fninf.2014.00003

Cardin, J. A., Carlén, M., Meletis, K., Knoblich, U., Zhang, F., Deisseroth, K., et al. (2009). Driving fast-spiking cells induces gamma rhythm and controls sensory responses. Nature 459, 663-667. doi: 10.1038/nature08002

Cohen, M. R., and Newsome, W. T. (2004). What electrical microstimulation has revealed about the neural basis of cognition. Curr. Opin. Neurobiol. 14, 169-177. doi: 10.1016/j.conb.2004.03.016

da Silva Pinto, M. A., de Souza, J. K. S., Baron, J., and Tierra-Criollo, C. J. (2011). A low-cost, portable, micro-controlled device for multi-channel LED visual stimulation. J. Neurosci. Methods 197, 82-91. doi: 10.1016/j.jneumeth.2011.02. 004

Englitz, B., David, S. V., Sorenson, M. D., and Shamma, S. A. (2013). MANTA-an open-source, high density electrophysiology recording suite for MATLAB. Front. Neural Circuits 7:69. doi: 10.3389/fncir.2013.00069

Flaherty, A., and Graybiel, A. M. (1994). Input-output organization of the sensorimotor striatum in the squirrel monkey. J. Neurosci. 14, 599-610.

Girardeau, G., Benchenane, K., Wiener, S. I., Buzsáki, G., and Zugaro, M. B. (2009). Selective suppression of hippocampal ripples impairs spatial memory. Nat. Neurosci. 12, 1222-1223. doi: 10.1038/nn.2384

Haikala, V., Joesch, M., Borst, A., and Mauss, A. S. (2013). Optogenetic control of fly optomotor responses. J. Neurosci. 33, 13927-13934. doi: 10.1523/JNEUROSCI. 0340-13.2013

Histed, M. H., Bonin, V., and Reid, R. C. (2009). Direct activation of sparse, distributed populations of cortical neurons by electrical microstimulation. Neuron 63, 508-522. doi: 10.1016/j.neuron.2009.07.016

Inagaki, H. K., Jung, Y., Hoopfer, E. D., Wong, A. M., Mishra, N., Lin, J. Y., et al. (2014). Optogenetic control of Drosophila using a red-shifted channelrhodopsin reveals experience-dependent influences on courtship. Nat. Methods 11, 325-332. doi: 10.1038/nmeth.2765

Kandel, E. R., Schwartz, J. H., and Jessell, T. M. (2000). Principles of Neural Science (Vol. 4). New York: McGraw-Hill.

Karlsson, M. P., and Frank, L. M. (2009). Awake replay of remote experiences in the hippocampus. Nat. Neurosci. 12, 913-918. doi: 10.1038/ nn. 2344

Klapoetke, N. C., Murata, Y., Kim, S. S., Pulver, S. R., Birdsey-Benson, A., Cho, Y. K., et al. (2014). Independent optical excitation of distinct neural populations. Nat. Methods 11, 338-346. doi: 10.1038/nmeth.2836

Kleiner, M., Brainard, D., Pelli, D., Ingling, A., Murray, R., and Broussard, C. (2007). What's new in Psychtoolbox-3. Perception 36, 1.1-1.16.

Newman, J. P., Zeller-Townson, R., Fong, M.-F., Desai, S. A., Gross, R. E., and Potter, S. M. (2013). Closed-loop, multichannel experimentation using the open-source NeuroRighter electrophysiology platform. Front. Neural Circuits 6:98. doi: 10. 3389/fncir.2012.00098 
Nikolic, K., Grossman, N., Grubb, M. S., Burrone, J., Toumazou, C., and Degenaar, P. (2009). Photocycles of Channelrhodopsin-2. Photochem. Photobiol. 85, 400411. doi: 10.1111/j.1751-1097.2008.00460.x

O'Connor, D. H., Hires, S. A., Guo, Z. V., Li, N., Yu, J., Sun, Q.-Q., et al. (2013). Neural coding during active somatosensation revealed using illusory touch. Nat. Neurosci. 16, 958-965. doi: 10.1038/nn.3419

O’Doherty, J. E., Lebedev, M. A., Hanson, T. L., Fitzsimmons, N. A., and Nicolelis, M. A. (2009). A brain-machine interface instructed by direct intracortical microstimulation. Front. Integr. Neurosci. 3:20. doi: 10.3389/neuro.07. 020.2009

Ohayon, S., Grimaldi, P., Schweers, N., and Tsao, D. Y. (2013). Saccade modulation by optical and electrical stimulation in the macaque frontal eye field. J. Neurosci. 33, 16684-16697. doi: 10.1523/JNEUROSCI.2675-13.2013

Pi, H.-J., Hangya, B., Kvitsiani, D., Sanders, J. I., Huang, Z. J., and Kepecs, A. (2013). Cortical interneurons that specialize in disinhibitory control. Nature 503, 521524. doi: 10.1038/nature12676

Pologruto, T. A., Sabatini, B. L., and Svoboda, K. (2003). ScanImage: flexible software for operating laser scanning microscopes. Biomed. Eng. Online 2:13. doi: 10.1186/1475-925X-2-13

Rolston, J. D., Gross, R. E., and Potter, S. M. (2009). A low-cost multielectrode system for data acquisition enabling real-time closed-loop processing with rapid recovery from stimulation artifacts. Front. Neuroeng. 2:12. doi: 10.3389/neuro. 16.012.2009

Sanders, J. I., and Kepecs, A. (2012). Choice ball: a response interface for psychometric discrimination in head-fixed mice. J. Neurophysiol. 108, 3416-3423. doi: 10. 1152/jn.00669.2012

Smear, M., Resulaj, A., Zhang, J., Bozza, T., and Rinberg, D. (2013). Multiple perceptible signals from a single olfactory glomerulus. Nat. Neurosci. 16, 16871691. doi: 10.1038/nn.3519

Soto-Faraco, S., Lyons, J., Gazzaniga, M., Spence, C., and Kingstone, A. (2002). The ventriloquist in motion: illusory capture of dynamic information across sensory modalities. Brain Res. Cogn. Brain Res. 14, 139-146. doi: 10.1016/s09266410(02)00068-x

Venkatraman, S., Elkabany, K., Long, J. D., Yao, Y., and Carmena, J. M. (2009). A system for neural recording and closed-loop intracortical microstimulation in awake rodents. IEEE Trans. Biomed. Eng. 56, 15-22. doi: 10.1109/TBME.2008. 2005944

Voigts, J., Siegle, J. H., Kemere, C., Moore, C. L., and Wilson, M. A. (2013a). “A lowcost, open-source system for combining high-channel count electrophysiology with closed-loop optogenetic feedback," in Paper Presented at the Society for Neuroscience (San Diego, CA).

Voigts, J., Siegle, J. H., Pritchett, D. L., and Moore, C. I. (2013b). The flexDrive: an ultra-light implant for optical control and highly parallel chronic recording of neuronal ensembles in freely moving mice. Front. Syst. Neurosci. 7:8. doi: 10. 3389/fnsys.2013.00008

Weick, J. P., Liu, Y., and Zhang, S.-C. (2011). Human embryonic stem cell-derived neurons adopt and regulate the activity of an established neural network. Proc. Natl. Acad. Sci. U S A 108, 20189-20194. doi: 10.1073/pnas.1108487108

Yamamoto, J., and Wilson, M. A. (2008). Large-scale chronically implantable precision motorized microdrive array for freely behaving animals. J. Neurophysiol. 100, 2430-2440. doi: 10.1152/jn.90687.2008

Zylberberg, A., Barttfeld, P., and Sigman, M. (2012). The construction of confidence in a perceptual decision. Front. Integr. Neurosci. 6:79. doi: 10.3389/fnint.2012. 00079

Conflict of Interest Statement: The authors declare that the research was conducted in the absence of any commercial or financial relationships that could be construed as a potential conflict of interest.

Received: 17 July 2014; accepted: 25 November 2014; published online: 11 December 2014.

Citation: Sanders JI and Kepecs A (2014) A low-cost programmable pulse generator for physiology and behavior. Front. Neuroeng. 7:43. doi: 10.3389/fneng.2014.00043 This article was submitted to the journal Frontiers in Neuroengineering.

Copyright (c) 2014 Sanders and Kepecs. This is an open-access article distributed under the terms of the Creative Commons Attribution License (CC BY). The use, distribution and reproduction in other forums is permitted, provided the original author(s) or licensor are credited and that the original publication in this journal is cited, in accordance with accepted academic practice. No use, distribution or reproduction is permitted which does not comply with these terms. 